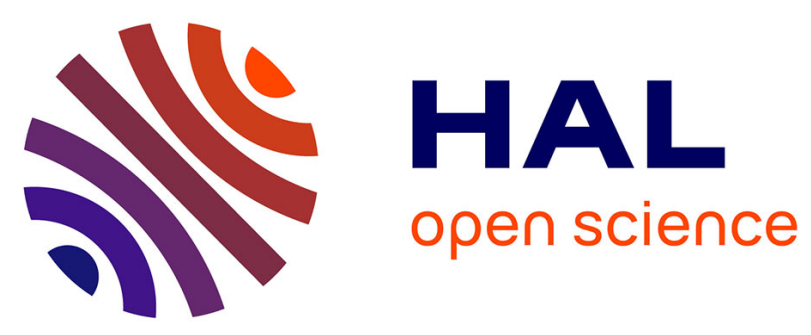

\title{
Examining the predictive relationship between personality and emotion traits and learners' agent-directed emotions
}

\author{
Jason M. Harley, Cassia K. Carter, Niki Papaioannou, François Bouchet, \\ Ronald S. Landis, Roger Azevedo, Lana Karabachian
}

\section{To cite this version:}

Jason M. Harley, Cassia K. Carter, Niki Papaioannou, François Bouchet, Ronald S. Landis, et al.. Examining the predictive relationship between personality and emotion traits and learners' agentdirected emotions. The 17th Conference on Artificial Intelligence in Education (AIED 2015), Jun 2015, Madrid, Spain. pp.145-154, 10.1007/978-3-319-19773-9_15 . hal-01340609

\section{HAL Id: hal-01340609 \\ https://hal.science/hal-01340609}

Submitted on 20 Mar 2019

HAL is a multi-disciplinary open access archive for the deposit and dissemination of scientific research documents, whether they are published or not. The documents may come from teaching and research institutions in France or abroad, or from public or private research centers.
L'archive ouverte pluridisciplinaire HAL, est destinée au dépôt et à la diffusion de documents scientifiques de niveau recherche, publiés ou non, émanant des établissements d'enseignement et de recherche français ou étrangers, des laboratoires publics ou privés. 


\title{
Examining the Predictive Relationship Between Personality and Emotion Traits and Learners' Agent-Direct Emotions
}

\author{
Jason M. Harley ${ }^{1,2}$, Cassia C. Carter ${ }^{3}$, Niki Papaionnou ${ }^{3}$, François Bouchet ${ }^{4,5}$, \\ Ronald S. Landis ${ }^{3}$, Roger Azevedo ${ }^{6}$, and Lana Karabachian ${ }^{2}$ \\ Université de Montréal, Computer Science and Operations Research, Montréal, QC, Canada ${ }^{1}$ \\ McGill University, Educational and Counselling Psychology, Montréal, QC, Canada ${ }^{2}$ \\ Illinois Institute of Technology, Psychology, Chicago, IL, USA ${ }^{3}$ \\ Sorbonne Universités, UPMC Univ. Paris 06, UMR 7606, LIP6, F-75005, Paris, France ${ }^{4}$ \\ CNRS, UMR 7606, LIP6, F-75005, Paris, France ${ }^{5}$ \\ North Carolina State University, Psychology, Raleigh, NC, USA ${ }^{6}$ \\ jason.umontreal.ca
}

\begin{abstract}
The current study examined the relationships between learners' $(N=$ 124) personality traits, the emotions they experience while typically studying (trait studying emotions), and the emotions they reported experiencing as a result of interacting with two Pedagogical Agents (PAs - agent-directed emotions) in MetaTutor, an advanced multi-agent learning environment. Overall, significant relationships between a subset of trait emotions (trait anger, trait anxiety) and personality traits (agreeableness, consciousness, and neuroticism) were found for four agent-directed emotions (pride, boredom, and neutral) though the relationships differed between the two PAs. These results demonstrate that some trait emotions and personality traits can be used to predict learners' emotions toward specific PAs (with different roles). Suggestions are provided for adapting PAs to support learners' (with certain characteristics) experience of positive emotions (e.g., enjoyment) and minimize their experience of negative emotions (e.g., boredom). Such an approach presents a scalable and easily implemented method for creating emotionally-adaptive, agent-based learning environments, and improving learner-PA interactions to support learning.
\end{abstract}

Keywords: Emotions, agent-directed emotions, trait emotions, personality traits, pedagogical agents, intelligent tutoring systems

\section{Introduction}

Emotions can critically impact how students learn with agent-based learning environments (ABLEs; [1-3]). ABLEs [4] are distinct from other computer-based learning environments (e.g., multi-agent systems, intelligent tutoring systems, serious games) because of their use of pedagogical agents (PAs); animated characters designed to provide several functions such as immediate and tailored prompts and feedback to support student learning. Despite research that examines emotions in these environments, very 
little is known in terms of why students experience different emotions during their interactions with these computer-based systems. For example, do students experience frustration because of the PA's feedback or because they are unable to locate multimedia material relevant to their current learning goal? Furthermore, there are contextual and individual difference variables, such as personality traits and trait emotions that can also contribute to the complexity of understanding the impact of emotions on learning with ABLEs. As such, the current study examined the relationships between learners' personality traits (five factor model; [4]), the emotions they experience while typically studying (trait studying emotions; [6]), and the emotions they reported experiencing as a result of the PAs (agent-directed emotions).

At present, research concerning students' agent-directed emotions is scarce. In the few existing studies, PAs have a range of different functions and features, such as deploying strategies to help students regulate their emotions, different dynamic facial expressions, gender, and race [1-4]. In addition to the characteristics of the PA, a number of learner characteristics (gender, prior knowledge, personality traits) have also been shown to influence learner-PA interactions $[1,2,8]$. However, the paucity of research on this topic makes any conclusions or recommendations tentative. This study contributes to the literature by evaluating the predictive utility of two sets of learner characteristics and how this information can be used to provide individually-tailored, user-system adaptation [8]. User-system adaptation involves changing features of the ABLE (e.g., PAs) to optimize them for different types of learners. In this study, MetaTutor, an ABLE for learning about the human circulatory system and fostering self-regulated learning (SRL) was used to examine learner-PA interactions.

\subsection{Learner characteristics}

Trait emotions. Trait emotions represent one set of individual differences that are related to what students typically experience while learning with respect to the behaviours they engage in while learning and their learning outcomes [9]. Specifically, trait emotions are habitual, re-occurring emotions experienced in a particular achievement context, such as feeling anxiety during tests or boredom while studying algebra. Pekrun [9] contrasts trait emotions with state emotions, which are emotions experienced in response to a particular situation (e.g., academic achievement situation). The relationship between trait studying emotions and agent-directed emotions as experienced during a studying task with an ABLE has not yet been examined. In addition to learners' trait emotions, personality traits are examined as a second source of individual differences.

Personality traits. Personality traits reflect individual differences in stable dispositions, which determine one's pattern of thought, emotionality, and behaviour $[5,10]$. Although several models of personality have been developed, the most comprehensive framework and consistent findings have derived from the five-factor model (FFM; [5]). These factors include Neuroticism (tendency to be temperamental and experience negative moods and feelings; e.g., Anxiety, and Depression), Conscientiousness (associated with efficiency, determination, responsibility, and persistence), Agreeableness 
(tendency to be more friendly, considerate of others, altruistic, sympathetic), Extraversion (associated with high physical and verbal activity, assertiveness, sociability), and Openness to Experience (tendency to prefer novel and broader ideas and experiences, intellectual activities, creativity). The FFM represents the highest level of the personality hierarchy, encompassing most of personality elements into five dimensions, thus providing order to various measures of personality. Numerous studies have empirically shown that personality is an important predictor of academic performance [10].

\subsection{The current study}

The purpose of the current study was to examine the relationship between learners' trait emotions as well as personality traits and their agent-directed emotions while interacting with MetaTutor. Given the novelty of the study, no suitable theoretical frameworks or corpus of prior research offered a compelling conceptual foundation upon which to formulate strong hypotheses. As a result, we identified our main research questions as follows: What are the predictive effects of personality traits and trait emotions on participants' agent-directed emotions (i.e., how participants reported the agents made them feel during the learning session), and does the quality of prompts and feedback provided by the PA (i.e., experimental condition) moderate these associations? Given space constraints, we report the results on two of the four PAs in MetaTutor, the ones that are the most consistently active throughout the learning session (see 2.2 and 2.3).

\section{Methods}

\subsection{Participants}

One hundred and twenty four undergraduate students from two North American Universities (one large, public institution and one small, private institution) participated in this study. Participants (65.3\% female; 64.5\% Caucasian) had a mean age of 21 and mean GPA of 3.07 and were randomly assigned to either an experimental or control condition (see 2.3 for details).

\subsection{MetaTutor Learning Environment}

MetaTutor was developed to teach students about the circulatory system and how to regulate their learning. MetaTutor is an adaptive, multi-agent hypermedia learning environment which presents 38 digital pages of human circulatory-system content (including text and diagrams) organized in a table of contents. The main interface of MetaTutor includes a timer that indicates how much time remains in the learning session, and an SRL palette where participants can initiate interactions with one of four PAs depending on the action chosen. Participants' subgoals are displayed during the learning session directly below their overall learning goal (to learn all they could about the human circulatory system; located in the upper-center portion of the screen) within progress bars automatically filled as learners navigate through pages relevant to the 
currently active subgoal. One of the four PAs is always visible in the upper right-hand corner of the learning environment. Each is responsible for specific tasks during the learning session, such as certain self-regulatory processes, and provides audible assistance through the use of a text-to-speech engine. Specifically, Gavin the Guide provides guidance for participants in the learning environment and administers pretest and posttest knowledge assessments and self-report measures, Pam the Planner prompts and scaffolds planning processes primarily at the beginning of the learning session (e.g., prior knowledge activation, setting subgoals), Mary the Monitor prompts and supports participants in their monitoring processes (e.g., judgment of learning), and Sam the Strategizer prompts participants to engage in learning strategies and ensures their use (e.g., note-taking, summarizing). Mary and Sam are the PAs examined in this study because they provide (1) interventions (2) throughout the learning session.

\subsection{Experimental design and learning conditions}

Participants were randomly assigned to either an experimental Prompt and Feedback (PF; $n=59)$ condition or Control $(n=65)$ condition. The strategies used in each condition differed in nature, detail, and amount of scaffolding provided by the PAs. Table 1 summarizes the two PA's behavior by condition that this paper examines.

Table 1. Summary of PA behaviour by condition and learning context

\begin{tabular}{|c|c|c|}
\hline Context & Summary of PA Behaviour & \\
\hline \multirow[b]{2}{*}{$\begin{array}{l}\text { Self-regulated } \\
\text { Learning } \\
\text { Behaviour }\end{array}$} & Prompt and Feedback Condition & Control Condition \\
\hline & $\begin{array}{l}\text { Prompts: Sam and Mary provide prompts to students to en- } \\
\text { gage in SRL behaviours, such as making summaries and } \\
\text { content evaluations. } \\
\text { Feedback: Sam and Mary provide feedback regarding stu- } \\
\text { dents' use of SRL processes. E.g. whether students have } \\
\text { written an appropriate summary; Mary agrees that the con- } \\
\text { tent of a page is relevant. Feedback irrespective of whether } \\
\text { they are prompted by the agent or self-initiated (through the } \\
\text { SRL palette) by the learner. }\end{array}$ & $\begin{array}{l}\text { Prompts: Sam and } \\
\text { Mary do not provide } \\
\text { any SRL prompts. } \\
\text { Feedback: Sam and } \\
\text { Mary do not provide } \\
\text { any feedback. }\end{array}$ \\
\hline $\begin{array}{l}\text { Administration of } \\
\text { assessments }\end{array}$ & $\begin{array}{l}\text { Mary administers page and subgoal quizzes to participants. } \\
\text { Provides feedback on how learners did on them; helps them } \\
\text { decide whether to move on or revisit learning material. }\end{array}$ & $\begin{array}{l}\text { Mary gives page and } \\
\text { subgoal quizzes. No } \\
\text { feedback provided. }\end{array}$ \\
\hline
\end{tabular}

\subsection{Experimental procedure}

The experiment consisted of two sessions (Session 1 lasted approximately 30 minutes and Session 2 lasted approximately three hours), separated by a maximum of three days or a minimum of one hour to avoid participant fatigue. During Session 1, participants read and signed an informed consent form and were administered several self-report questionnaires (e.g., demographics, mini-IPIP, AEQ-trait emotions - cf. section 2.5) and a content pretest. All participants completed the session individually on a desktop computer. Participants were compensated up to $\$ 10$ at the end of session 1 (\$10/hr.) or given course credit (depending on availability of a subject pool). 
During session 2, participants were shown an introductory video presenting information regarding the MetaTutor learning environment, including training on how to use different components and navigate through the system. Participants were also provided with instructions on how to set and create their own subgoals. Each participant was given an hour to learn as much as possible about the circulatory system using MetaTutor. Halfway through the learning session, participants were given the opportunity to take a five-minute break. At the end of session 2, participants were asked to complete a posttest followed by a series of self-report questionnaires. Lastly, all participants were debriefed and received up to $\$ 40$ for participating in the study.

\subsection{Measures and materials}

Achievement Emotions Questionnaire (AEQ-Trait). The AEQ [6] was used to assess the cognitive, motivational, and physiological components of emotions. The measure consists of 24 scales that assess class-related, learning-related, and test-related emotions. Only the 75-item learning-related emotions scale, consisting of eight learningrelated emotions (Enjoyment, Hope, Pride, Anger, Shame, Anxiety, Hopelessness, and Boredom) was used in the current study. Items were grouped into three sections that assessed how participants usually felt before, during, and after studying in an academic setting. The AEQ uses a 5-point Likert response scale, ranging from 1 (strongly disagree) to 5 (strongly agree). A sample item from the before studying section is "I look forward to studying." A mean score was derived for each learning-related trait emotion. Coefficient alphas for the learning-related scales ranged from .53 to .93.

Mini-International Personality Item Pool (mini-IPIP). The mini-IPIP is a 20-item version of the 50-item International Personality Item Pool-Five-Factor Model measure. The mini-IPIP [11] was used to assess the personality dimensions of the FFM. Participants were asked to respond to each item using a 5-point Likert response scale ranging from 1 (very inaccurate) to 5 (very accurate). A sample item is "I get upset easily." Mean scores were generated for each personality dimension. This measure demonstrated adequate internal consistencies for all dimensions $(\alpha=.71-.77)$.

Agent Response Inventory (ARI). The ARI consists of 76 items assessing the extent to which each of the four PAs made them feel 19 different discrete emotions (Happiness, Enjoyment, Hope, Pride, Curiosity, Eureka, Anger, Frustration, Anxiety, Shame, Fear, Contempt, Disgust, Boredom, Hopelessness, Sadness, Surprise, Confusion, and Neutral). Participants were asked to respond to each item using a 5-point Likert response scale ranging from 1 (strongly disagree) to 5 (strongly agree). A sample item was "SAM made me feel that I was enjoying myself." The ARI and the digital definition handout (provided in a side panel to participants while they filled out the self-report) were based on a similar, single-item self-report measure that had good agreement rates with emotions obtained from automatic facial expression recognition software (the emotion-value questionnaire: EV; [7]). 


\section{$3 \quad$ Results}

\subsection{Data cleaning and descriptive statistics}

Prior to addressing our research questions, we first examined all of our predictor and dependent variables for skewness, kurtosis, and outliers. There were a large number of highly skewed variables, especially in the ARI emotions for various agents. Many of the negative emotions, such as Disgust, Frustration, or Anger, were not elicited by any of the agents and were removed from subsequent analyses (which does not mean learners never experienced these emotions, as seen in other studies [7], only that they did not identify the agents as the cause of those emotions). We set the threshold for identifying significant skew or kurtosis at $z=+/-3.35$.

Descriptive statistics for the personality scales, the trait emotion scales, and the agent-directed emotions are reported in Tables 2 and 3 (skewed variables that were not included in further analyses are italicized). The AEQ trait emotion means ranged from 2.01 to 3.93 on a five-point scale. The mini-IPIP scale means ranged from 2.74 to 4.06 on a five-point scale. The ARI emotions that were left as dependent variables were Happy, Enjoyment, Hopeful, Proud, Bored, and Neutral for both agents. The emotion of Curiosity was also analyzed for Mary, but not Sam. The means on these variables ranged from 2.28 to 3.44 on a 1-5 scale.

\subsection{Personality traits, trait emotions, condition, and agent-directed emotions}

The research question was evaluated with a series of moderated regression analyses, one for Sam and one for Mary, which tested for the presence of significant effects of two sets of predictor variables (personality traits and trait emotions) on agent-directed emotions while considering the moderating effect of Condition. For each of the moderated multiple regression analyses, the predictor variables, along with Condition, were entered as main effects into the first step while the variables and their interactions with Condition were entered into the second step. Higher-order (e.g., 3-way) interaction effects were not included. These regressions were run for each ARI emotion for each agent. Results for significant models are discussed below (cf. Tables 2 and 3).

Sam the Strategizer. We examined whether participants felt more or less Happiness, Enjoyment, Hopefulness, Pride, Boredom, or Neutrality due to their interactions with him. The model for Step 1 of the moderated regression on Happiness was statistically significant $\left(R^{2}=.20, p<.05\right)$. Specifically, a statistically significant main effect was found for Condition $(\beta=-0.30, p<.01)$, such that participants in the Control condition experienced higher levels of Happiness with Sam. However, the change in $R^{2}$ was not statistically significant for the model of the second step, and there were no statistically significant interactions.

For Neutral emotion expression as the outcome measure, the models for both step 1 $\left(R^{2}=.19, p<.05\right)$ and step $2\left(R^{2}=.33, p<.01\right)$ were statistically significant. A main effect was found for Condition $(\beta=-0.33, p<.01)$, where participants in the Control condition felt significantly more Neutral towards Sam. Interaction effects were found 
for Anxiety and Condition $(\beta=-0.54, p<.05)$, Anger and Condition $(\beta=0.53, \mathrm{p}<.01)$, and Conscientiousness and Condition $(\beta=-0.24, p<.05)$.

Table 2. Descriptive Stats. for AEQ and IPIP

\begin{tabular}{llrr}
\hline \multicolumn{2}{c}{ Variables } & $M$ & $S D$ \\
\hline \multirow{4}{*}{ Shame } & 2.63 & 0.87 \\
& Anxious & 2.93 & 0.80 \\
& Anger & 2.26 & 0.70 \\
AEQ & Pride & 3.93 & 0.60 \\
& Hope & 3.67 & 0.69 \\
& Enjoyment & 3.52 & 0.55 \\
& Bored & 2.58 & 0.85 \\
& Hopelessness & 2.01 & 0.82 \\
& & & \\
& Agreeableness & 4.06 & 0.63 \\
& Conscientious- & 3.74 & 0.76 \\
& ness & & \\
ExIP & & \\
Extraversion & 3.21 & 0.89 \\
& Openness & 3.88 & 0.75 \\
& Neuroticism & 2.74 & 0.85 \\
\hline
\end{tabular}

Table 3. Descriptive Stats. for ARI

\begin{tabular}{lrrrr}
\hline \multirow{2}{*}{ ARI Emotions } & \multicolumn{2}{c}{ Sam } & \multicolumn{2}{c}{ Mary } \\
& $M$ & $S D$ & $M$ & $S D$ \\
\hline happiness & 2.69 & 1.06 & 3.00 & 1.10 \\
enjoyment & 2.49 & 1.07 & 2.76 & 1.16 \\
hopefulness & 2.71 & 1.07 & 2.96 & 1.18 \\
pride & 2.56 & 1.07 & 2.76 & 1.18 \\
anger & 1.97 & 1.30 & 1.89 & 1.20 \\
frustration & 2.14 & 1.38 & 2.05 & 1.25 \\
anxiety & 1.95 & 1.25 & 2.08 & 1.28 \\
fear & 1.48 & 0.86 & 1.53 & 0.95 \\
shame & 1.55 & 0.97 & 1.54 & 0.91 \\
hopelessness & 1.51 & 0.94 & 1.49 & 0.86 \\
boredom & 2.49 & 1.29 & 2.37 & 1.27 \\
surprise & 1.85 & 1.15 & 1.93 & 1.19 \\
contempt & 2.02 & 1.31 & 1.93 & 1.20 \\
disgust & 1.26 & 0.63 & 1.21 & 0.59 \\
confusion & 1.81 & 1.24 & 1.65 & 0.95 \\
curiosity & 1.98 & 1.15 & 2.28 & 1.31 \\
sadness & 1.29 & 0.66 & 1.27 & 0.62 \\
eureka & 1.46 & 0.87 & 1.80 & 1.18 \\
neutral & 3.25 & 1.43 & 3.24 & 1.34 \\
\hline
\end{tabular}

Note. Variables in italics were beyond the skewness cutoff and were not used in further analyses

Examining the visual representation of the significant interactions for Sam, Figure 1 shows that participants in the Control condition with lower levels of trait Anger experienced lower levels of Neutral feelings than those with higher levels of trait Anger. In contrast, participants in the PF condition mirrored these differences, but at lower levels of Neutral elicited from Sam. Figure 2 shows a pattern of interaction results with Condition moderating the effects of trait Anxiety on the Neutral emotion outcome. Participants in the Control condition experienced more Neutral feelings at higher trait Anxiety levels, where participants in the PF condition experienced more Neutral feelings at lower Anxiety levels. The moderation of Condition and Conscientiousness for Sam predicting Neutral follows the same pattern as Figure 2. Participants in the Control condition experienced more Neutral feelings toward Sam when they had high levels of Conscientiousness, whereas participants in the PF condition experienced more Neutral feelings toward Sam when they had low levels of Conscientiousness.

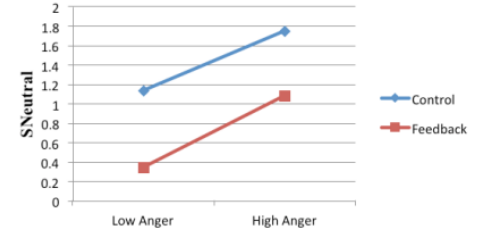

Fig.1. Interaction results for Anger and Condition predicting Neutral for Sam.

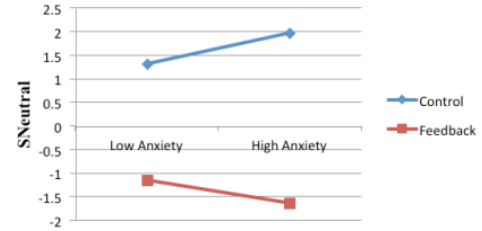

Fig.2. Interaction results for Anxiety and Condition predicting Neutral for Sam. 
Mary the Monitor. The criterion of Pride yielded statistically significant results. The model for Step $1\left(R^{2}=.21, p<.01\right)$ showed a statistically significant main effect of Condition $(\beta=0.25, p<.01)$, such that participants in the PF condition experienced significantly more feelings of Pride with Mary. The model for Step $2\left(R^{2}=.35, p<.01\right)$ yielded a statistically significant interaction of Anger and Condition $(\beta=-0.48, p<.05)$ and of Conscientiousness and Condition $(\beta=-0.29 p<.05)$. Figure 2 shows the pattern of interaction results for both of these interactions. For the Anger and Condition interaction, participants in the Control condition experienced higher levels of Pride with Mary at higher levels of trait Anger, whereas participants in the PF condition experienced higher levels of Pride at lower levels of trait Anger. For the Conscientiousness interaction with Condition, participants in the Control condition reported higher levels of Pride for higher levels of Conscientiousness; participants in the PF condition experienced higher levels of Pride for lower levels of Conscientiousness.

The analyses on Boredom did not have a statistically significant model for Step 1, but the model for Step 2 was statistically significant $\left(R^{2}=.30, p<.05\right)$. Specifically, the model from Step 2 yielded a statistically significant main effects for Neuroticism $(\beta$ $=0.50, p<.05)$ and Condition $(\beta=-0.22, p<.05)$, and a statistically significant interaction between Neuroticism and Condition $(\beta=-0.44, p<.01)$. Figure 2 shows the graph of the pattern of this interaction. Participants in the Control condition reported higher levels of Boredom at higher levels of Neuroticism and participants in the PF condition reported higher levels of Boredom when they had low Neuroticism.

\section{Discussion}

\subsection{Sam}

Our results revealed that participants were more likely to feel more Neutral toward Sam in the Control condition. Students in the PF condition were more likely to experience lower mean levels of Neutral toward Sam than in the Control condition, in particular those low in Trait Anger. Given that Neutral is assumed to be an adaptive state in which learners are not distracted by negative emotions, such as Boredom or Frustration, it can be seen as an appropriate agent-directed emotional classification, albeit not as adaptive as the positively-valenced, activating emotions of Enjoyment and Curiosity. Therefore, our results for Sam indicate an area for further examination, specifically to see what emotions students in the PF condition, in particular those low in trait Anger, experienced instead of Neutral. A preliminary review of the differences in the means between agent-directed emotions indicates that learners experienced higher levels of negative activating emotions, such as Anxiety and Frustration, in the PF condition than in the Control condition. It is possible that this could be due to certain prompts (e.g., mandatory summaries) that Sam gives which some students (e.g., those low in trait anger) may find disruptive rather than helpful. Therefore, if students are identified as being low in trait anger the system could reduce the number of prompts Sam provides to help minimize learners' experience of negative emotions. 


\subsection{Mary}

Results revealed that students high in Agreeableness were more likely to feel Proud when interacting with Mary and those high in Neuroticism were more likely to feel more Bored. When considering Condition, learners were more likely to report feeling more Proud and less Bored in terms of their interactions with Mary when in the PF condition (and vice versa for the Control condition). Students high in trait Anger and Conscientiousness were more likely to experience higher levels of Pride when interacting with Mary in the Control condition, while students high in Neuroticism were more likely to feel Bored on account of Mary in the Control condition. These results suggest that Mary's interactions with students elicited the most positive responses from students in the PF condition, where she helped students monitor their learning rather than play a passive role until they self-initiated monitoring behaviors. They may have also felt more proud in the PF condition on account of Mary congratulating them when they accurately assessed their level of knowledge regarding a page of content. It is possible that students who are used to experiencing Anger while studying may have felt Proud and considered Mary partially responsible for this emotion, even though her role was minimal in the Control condition. Students high in Conscientiousness may have felt that Mary gave them more autonomy in the Control condition and therefore felt more Pride.

A preliminary review of the differences in frequencies between students' use of selfvs. PA-initiated SRL behaviors indicates that learners in the Control condition engaged in more self-initiated SRL behavior than learners in the PF condition. Therefore, if students were identified as being high in consciousness, they may benefit from fewer prompts by experiencing more Pride, in addition to engaging in more self-initiated SRL behaviors. Although students high in Neuroticism were more Bored with Mary in the Control condition, Mary's interactions with them were minimal and informational at most when they didn't self-initiate monitoring behaviors. Therefore, more Neurotic students may have reported that Mary made them feel Bored in the Control condition if they were Bored to begin with in MetaTutor, and therefore expected her to interact with them more. Therefore, it is not necessary to adapt behavior.

\subsection{Conclusions, Limitations, and Future Directions}

This study examined the predictive effects of learners' trait emotions and personality traits on agent-directed emotions. Overall, significant relationships between a subset of trait emotions and personality traits were found, though the relationships differed between PAs. These results demonstrate that some trait emotions (trait Anger and trait Anxiety) and personality traits (Agreeableness, Conscientiousness, and Neuroticism) can be used to predict learners' agent-directed emotions toward specific PAs (Pride, Boredom, and Neutral). The skewed distribution of the majority of the agent-directed emotion measures (low levels) was a limitation of this study. Low levels of emotions and higher levels of Neutral have, however, been previously identified with other measures and studies with MetaTutor. The size of our sample is also a limitation of this study, which resulted in weaker statistical power than would have been ideal for the 
number of predictive variables examined. This study is novel in exploring these affective relationships, however, further research is required to draw conclusions regarding the relationship between agent-directed emotions, trait emotions, personality traits, and learning. More research is also needed in order to make further instructional prescriptions for PAs regarding how their prompts and feedback can be adapted to learners' individual characteristics, though this study provides a number of insights. Such an approach could present a scalable and easily implemented means for creating more emotionally-adaptive ABLEs and improving learner-PA interactions.

Acknowledgements. The research presented in this paper has been supported by a doctoral and postdoctoral fellowship from the Fonds Québécois de recherche - Société et culture (FQRSC) and a Joseph-Armand Bombardier Canada Graduate Scholarship for Doctoral research from the Social Sciences and Humanities Research Council (SSHRC) of Canada awarded to the first author. This research has also been supported by funding awarded to the sixth author from the National Science Foundation (IIS 1008282), SSHRC, and the Canada Research Chairs program.

\section{References}

1. D'Mello, S., \& Graesser, A. AutoTutor and Affective Autotutor. ACM Transactions on Interactive Intelligent Systems, 2(4), 1-39. (2013).

2. Arroyo, I., Woolf, B. P., Royer, J. M., \& Tai, M. Affective gendered learning companions. Artificial Intelligence in Education (pp. 41-48). Amsterdam: IOS Press. (2009).

3. Conati, C., \& Maclaren, H. Empirically building and evaluating a probabilistic model of user affect. User Modeling and User-Adapted Interaction, 19, 267-303. (2009).

4. Harley, J. M., \& Azevedo, R. Understanding students' emotions during interactions with advanced agent-based learning environments. ITS (pp. 629-631). Berlin: Springer. (2014).

5. Costa, P. T., \& McCrae, R. R. (1992). Normal personality assessment in clinical practice: The NEO Personality Inventory. Psychological Assessment, 4(1), 5-13.

6. Pekrun, R., Goetz, T., Titz, W., \& Perry, R. Academic achievement emotions in students' self-regulated learning and achievement. Educational Psychologist, 37, 91-206. (2002).

7. Harley, J. M., Bouchet, F., Hussain, S., Azevedo, R., \& Calvo, R. A multi-componential analysis of emotions during complex learning with an intelligent multi-agent system. Computers in Human Behavior, 48, 615-625. (2015).

8. Sabourin, J., Mott, B., \& Lester, J. C. (Modeling learner affect with theoretically grounded dynamic Bayesian networks. ACII (pp. 286-295). Berlin: Springer. (2011).

9. Pekrun, R. (2006). The control-value theory of achievement emotions: assumptions, corollaries, and implications for ed. research and practice. Ed. Psych. Review, 18, 315-341.

10. Chamorro-Premuzic, T., \& Furnham, A. (2005). Personality and intellectual competence. Mahwah, NJ: Erlbaum.

11. Donnellan, M. B., Oswald, F. L., Baird, B. M., \& Lucas, R. E. The mini-IPIP scales. Psychological Assessment, 18, 192-203. (2006). 